\title{
The periodic version of the Da Prato-Grisvard theorem and applications to the bidomain equations with FitzHugh-Nagumo transport
}

\author{
Matthias Hieber $^{1} \cdot$ Naoto Kajiwara ${ }^{2} \cdot$ Klaus Kress $^{1}$ • Patrick Tolksdorf ${ }^{3}$
}

Received: 21 February 2020 / Accepted: 13 March 2020 / Published online: 30 March 2020

(c) The Author(s) 2020

\begin{abstract}
In this article, the periodic version of the classical Da Prato-Grisvard theorem on maximal $L^{p}$-regularity in real interpolation spaces is developed, as well as its extension to semilinear evolution equations. Applying this technique to the bidomain equations subject to ionic transport described by the models of FitzHugh-Nagumo, Aliev-Panfilov, or RogersMcCulloch, it is proved that this set of equations admits a unique, strong T-periodic solution in a neighborhood of stable equilibrium points provided it is innervated by $T$-periodic forces.
\end{abstract}

Keywords Maximal regularity in real interpolation spaces - Theorem of Da Prato and Grisvard · Periodic solutions to semilinear evolution equations $\cdot$ Bidomain system

Mathematics Subject Classification 35B10 $\cdot 92 \mathrm{C} 35 \cdot 35 \mathrm{~K} 58$

Naoto Kajiwara was supported by JSPS Japanese-German Graduate Externship and scientific grant Kiban S (26220702) as a research assistant of the University of Tokyo. Klaus Kress and Patrick Tolksdorf were supported by the DFG International Research Training Group 1529 on Mathematical Fluid Dynamics at TU Darmstadt.

Patrick Tolksdorf

tolksdorf@uni-mainz.de

Matthias Hieber

hieber@mathematik.tu-darmstadt.de

Naoto Kajiwara

kajiwara_naoto@ma.noda.tus.ac.jp

Klaus Kress

kkress@mathematik.tu-darmstadt.de

1 Fachbereich Mathematik, Technische Universität Darmstadt, Schlossgartenstr. 7, 64289 Darmstadt, Germany

2 Department of Mathematics, Faculty of Science and Technology, Tokyo University of Science, Noda, Chiba 278-8510, Japan

3 Institut für Mathematik, Johannes Gutenberg-Universität Mainz, Staudingerweg 9, 55099 Mainz, Germany 


\section{Introduction}

Given a sectorial operator $\mathcal{A}$ on a Banach space $X$, the problem of maximal regularity for the inhomogenous Cauchy problem

$$
\left\{\begin{array}{l}
u^{\prime}(t)+\mathcal{A} u(t)=f(t), \quad 0<t<T \\
u(0)=0
\end{array}\right.
$$

received a lot of attention since the pioneering articles by Da Prato-Grisvard [10], Dore-Venni [13] and Lunardi [22]. Nowadays, due to a result due to Weis [26], it is well known that for $X$ being a UMD-space and $1<p<\infty$, maximal $L^{p}$-regularity of (ACP) can be characterized in terms of the $\mathcal{R}$-boundedness of the resolvent of $\mathcal{A}$. For further information, see also[11, 12] and [23].

The aim of this article is twofold: we first study maximal regularity of the inhomogeneous Cauchy problem within the periodic setting

$$
\begin{cases}u^{\prime}(t)+\mathcal{A} u(t)=f(t), & t \in \mathbb{R}, \\ u(t)=u(t+T), & t \in \mathbb{R}\end{cases}
$$

as well as for its semilinear counterpart. The results are then applied to the bidomain equations with FitzHugh-Nagumo transport. This is a well-established system of equations describing the electrical activities of the heart, see, e.g., Colli Franzone et al. [7] and the monograph by Keener and Sneyd [21].

Let us start by describing the problem of maximal periodic regularity for the period $T=2 \pi$. We say that $\mathcal{A}$ admits maximal periodic $L^{p}$-regularity, if for all $f \in L^{p}(0,2 \pi ; X)$ Eq. (PACP) with $T=2 \pi$ admits a unique solution $u$ within the class

$$
u \in \mathrm{W}^{1, p}(0,2 \pi ; X) \cap L^{p}(0,2 \pi ; D(\mathcal{A})) .
$$

It was proved by Arendt-Bu [4] that, given $1<p<\infty, X$ a UMD-space, and $\mathcal{A}: D(\mathcal{A}) \rightarrow X$ a closed operator, $\mathcal{A}$ admits maximal periodic $L^{p}$-regularity if and only if

$$
\mathrm{i} \mathbb{Z} \subset \varrho(\mathcal{A}) \quad \text { and } \quad k(\mathrm{i} k-\mathcal{A})^{-1} \text { is } \mathcal{R} \text {-bounded. }
$$

For definitions of UMD-spaces and $\mathcal{R}$-bounded families of operators as well as their properties, we refer, e.g., to [11] or [20].

In this article, we have the following two extensions of this result in mind: The first is, that some situations make it necessary to handle the case $p=1$. However, it is known that if maximal regularity holds for (ACP) for $p=1$, then $X$ is necessarily a non-reflexive space and that contradicts the UMD-property. The second extension we have in mind is to provide an elegant way to treat polynomial nonlinearities. These can be estimated easily if the ground space $X$ is a Banach algebra. The largest Banach algebra within the scale of Sobolev spaces is the Besov space $\mathrm{B}_{p, 1}^{n / p}$, which is again not reflexive and thus does not enjoy the UMD-property. In the classical maximal regularity theory for (ACP), a result accounting for these issues is the well-known theorem of Da Prato and Grisvard. In fact, let $-\mathcal{A}$ be the generator of a bounded analytic semigroup $\mathrm{e}^{-t \mathcal{A}}$ on a Banach space $X$ with domain $D(\mathcal{A})$. For $\theta \in(0,1)$ and $1 \leq p<\infty$, we denote by $D_{\mathcal{A}}(\theta, p)$ the space defined as

$$
D_{\mathcal{A}}(\theta, p):=\left\{x \in X:[x]_{\theta, p}:=\left(\int_{0}^{\infty}\left\|t^{1-\theta} \mathcal{A} \mathrm{e}^{-t \mathcal{A}} x\right\|_{X}^{p} \frac{\mathrm{d} t}{t}\right)^{1 / p}<\infty\right\} .
$$


When equipped with the norm $\|x\|_{\theta, p}:=\|x\|+[x]_{\theta, p}$, the space $D_{\mathcal{A}}(\theta, p)$ becomes a Banach space. It is well known that $D_{\mathcal{A}}(\theta, p)$ coincides with the real interpolation space $(X, D(\mathcal{A}))_{\theta, p}$ and that the respective norms are equivalent. If $0 \in \rho(\mathcal{A})$, then the real interpolation space norm is equivalent to the homogeneous norm $[\cdot]_{\theta, p}$, see [17, Corollary 6.5.5].

Let now $\theta \in(0,1), 1 \leq p<\infty$, and $0<T<\infty$. Then, for $f \in L^{p}\left(0, T ; D_{\mathcal{A}}(\theta, p)\right)$ we consider

$$
u(t):=\int_{0}^{t} \mathrm{e}^{-(t-s) \mathcal{A}} f(s) \mathrm{d} s, \quad 0<t<T,
$$

which is the unique mild solution to the abstract Cauchy problem (ACP) and fulfills, thanks to the classical Da Prato and Grisvard theorem [10], the following maximal regularity estimate.

Theorem 1.1 ([10, Da Prato-Grisvard]) Let $\theta \in(0,1), 1 \leq p<\infty$, and $0<T<\infty$. Then there exists a constant $C>0$ such that for all $f \in L^{p}\left(0, T ; D_{\mathcal{A}}(\theta, p)\right)$, the function $u$ given by (1.2) satisfies $u(t) \in D(\mathcal{A})$ for almost every $0<t<T$ and

$$
\|\mathcal{A} u\|_{L^{p}\left(0, T ; D_{\mathcal{A}}(\theta, p)\right)} \leq C\|f\|_{L^{p}\left(0, T ; D_{\mathcal{A}}(\theta, p)\right)} .
$$

As counterpart to the Arendt-Bu theorem characterizing maximal periodic $L^{p}$-regularity for $1<p<\infty$ and $X$ being a UMD-space, we provide a short derivation of a periodic version of Theorem 1.1. As the classical Da Prato-Grisvard theorem, it holds for $1 \leq p<\infty$ and arbitrary Banach spaces $X$.

To formulate this result, we define the periodicity of a measurable function as follows. For $0<T<\infty$, we call a measurable function $f: \mathbb{R} \rightarrow X$ periodic of period $T$ if $f(t)=f(t+T)$ holds true for almost all $t \in(-\infty, \infty)$. Then, formally, a candidate for a solution $u$ to (PACP) is given by

$$
u(t):=\int_{-\infty}^{t} \mathrm{e}^{-(t-s) \mathcal{A}} f(s) \mathrm{d} s .
$$

The periodic version of the Da Prato-Grisvard theorem now states that, under certain assumptions on $\mathcal{A}$ and $f, u$ is indeed well-defined, continuous, and periodic and satisfies a maximal regularity estimate in the sense of Da Prato-Grisvard. More precisely, let $-\mathcal{A}$ be the generator of a bounded analytic semigroup on $X$ with $0 \in \rho(\mathcal{A})$, let $\theta \in(0,1)$, $1 \leq p<\infty$, and $0<T<\infty$. We then show that there exists a constant $C>0$ such that for all $T$-periodic functions $f: \mathbb{R} \rightarrow D_{\mathcal{A}}(\theta, p)$ with $f_{\mid(0, T)} \in L^{p}\left(0, T ; D_{\mathcal{A}}(\theta, p)\right)$ the function $u$ defined by (1.3) lies in $C\left(\mathbb{R} ; D_{\mathcal{A}}(\theta, p)\right)$, is periodic of period $T$, satisfies $u(t) \in D(\mathcal{A})$ for almost every $t \in \mathbb{R}$, and satisfies

$$
\|\mathcal{A} u\|_{L^{p}\left(0, T ; D_{\mathcal{A}}(\theta, p)\right)} \leq C\|f\|_{L^{p}\left(0, T ; D_{\mathcal{A}}(\theta, p)\right)} .
$$

Our second aim is to apply the above technique to the bidomain equations with FitzHugh-Nagumo (or related) transport terms as well as to the Allen-Cahn equations. Note that these equations are, however, semilinear evolution equations. Hence, in order to follow the above strategy, we first need to develop a semilinear version of the periodic $\mathrm{Da}$ Prato-Grisvard theorem. This will be done in Sect. 3.

The bidomain system is given by 


$$
\begin{cases}\partial_{t} u+F(u, w)-\nabla \cdot\left(\sigma_{\mathrm{i}} \nabla u_{\mathrm{i}}\right)=I_{\mathrm{i}} & \text { in }(0, \infty) \times \Omega, \\ \partial_{t} u+F(u, w)+\nabla \cdot\left(\sigma_{\mathrm{e}} \nabla u_{\mathrm{e}}\right)=-I_{\mathrm{e}} & \text { in }(0, \infty) \times \Omega, \\ \partial_{t} w+G(u, w)=0 & \text { in }(0, \infty) \times \Omega,\end{cases}
$$

subject to the boundary conditions

$$
\sigma_{\mathrm{i}} \nabla u_{\mathrm{i}} \cdot v=0, \quad \sigma_{\mathrm{e}} \nabla u_{\mathrm{e}} \cdot v=0 \quad \text { on } \quad(0, \infty) \times \partial \Omega,
$$

and the initial data

$$
u(0)=u_{0}, \quad w(0)=w_{0} \quad \text { in } \quad \Omega .
$$

Here $\Omega \subset \mathbb{R}^{n}$ denotes a domain describing the myocardium, the functions $u_{\mathrm{i}}$ and $u_{\mathrm{e}}$ model the intra- and extracellular electric potentials, $u:=u_{\mathrm{i}}-u_{\mathrm{e}}$ denotes the transmembrane potential, and $v$ denotes the outward unit normal vector to $\partial \Omega$. The anisotropic properties of the intra- and extracellular tissue parts will be described by the conductivity matrices $\sigma_{\mathrm{i}}(x)$ and $\sigma_{\mathrm{e}}(x)$. Furthermore, $I_{\mathrm{i}}$ and $I_{\mathrm{e}}$ stand for the intra- and extracellular stimulation current, respectively. The variable $w$, the so-called gating variable, corresponds to the ionic transport through the cell membrane.

Mathematical models describing the propagation of impulses in electrophysiology have a long tradition starting with the classical model by Hodgkin and Huxley in the 1950s, see, e.g., $[1,3,8,14,24]$ and the recent survey article of Stevens [25]. In this article, we consider the bidomain model subject to various models for the ionic transport including the models by FitzHugh-Nagumo, Aliev-Panfilov, and Rogers-McCulloch. The FitzHugh-Nagumo model reads as

$$
\begin{aligned}
& F(u, w)=u(u-a)(u-1)+w=u^{3}-(a+1) u^{2}+a u+w, \\
& G(u, w)=b w-c u,
\end{aligned}
$$

where $0<a<1$ and $b, c>0$. The Aliev-Panfilov model as well as the Rogers-McCulloch model are described in detail in Sect. 4.

Note that the so-called bidomain operator is a very non-local operator, which makes the analysis of this equation seriously more complicated compared, e.g., to the classical Allen-Cahn equation.

The rigorous mathematical analysis of this system started with the work of Colli Franzone and Savaré [9], who introduced a variational formulation of the problem and showed the global existence and uniqueness of weak and strong solutions for the FitzHugh-Nagumo model. In 2009, a new approach to this system was presented by Bourgault, Coudière, and Pierre in [6]. They introduced for the first time the so-called bidomain operator $A$ within the $L^{2}$-setting and showed that it is a nonnegative and self-adjoint operator. They further showed the existence and uniqueness of a local strong solution and the existence of a global, weak solution to the system above for a large class of ionic models.

New impetus to the field was recently given by Giga and Kajiwara [15], who investigated the bidomain equations within the $L^{p}$-setting for $1<p \leq \infty$. They showed that the negative of bidomain operator $A$ is the generator of an analytic semigroup on $L^{p}(\Omega)$ for $p \in(1, \infty]$ and constructed a local, strong solution to the bidomain system within this setting.

Very recently the bidomain operator $A$ was shown to admit a bounded $\mathrm{H}^{\infty}$-calculus on $L_{0}^{p}(\Omega)$, see [19]. Moreover, using the theory of critical spaces, it was shown in [18] that the 
bidomain equations admit global, strong solutions for $d=2,3$ for initial data lying in critical Besov spaces.

In this context, it is now a natural question to ask, whether the bidomain equations admit time-periodic solutions. Of course, periodic solutions can be formulated in various ways and various regularity classes, ranging from weak over mild to strong solutions. In this article, we are interested in unique, strong periodic solutions. We show that the innervated system admits a unique, strong time-periodic solution of period $T$ provided the outer forces $I_{\mathrm{i}}$ and $I_{\mathrm{e}}$ are both time-periodic of period $T>0$. Our approach to the existence of unique, strong $T$-periodic solutions to the bidomain equations in a neighborhood of stable equilibrium points is the semilinear version of the periodic $\mathrm{Da}$ Prato-Grisvard theorem in Sect. 3.

Let us emphasize that we consider here the full bidomain model taking into account the anisotropic phenomena and not only the so-called monodomain approximation.

Recently, a very different approach to periodic solutions to the bidomain equations was developed by Giga, Kajiwara, and Kress [16]. They showed the existence of a strong, periodic solution to the bidomain equations for arbitrary large $f \in L^{2}(\Omega)$ based on a weak-strong uniqueness argument. Observe, however, that this approach does, by its nature, not yield uniqueness of the periodic solution. On the other hand, the semilinear version of the periodic Da Prato-Grisvard theorem given in Sect. 3 yields existence and uniqueness of a periodic solution and allows also to consider forcing terms $f \in L^{p}\left(0, T ; D_{A}(\theta, p)\right)$.

This paper is organized as follows: Sect. 2 presents the periodic version of the Da Prato-Grisvard theorem, which will be extended in Sect. 3 to the semilinear setting. In Sect. 4, we present our second main result, the existence of a unique, strong $T$-periodic solution to the bidomain equations subject to a large class of models for the ionic transport. The proofs of the main results concerning the bidomain equations will be given in Sect. 5.

\section{A periodic version of the Da Prato-Grisvard theorem}

Let $X$ be a Banach space and $-\mathcal{A}$ be the generator of a bounded analytic semigroup on $X$, i.e., $\mathcal{A}$ is closed and densely defined and there exists $\theta \in(\pi / 2, \pi)$ and $C>0$ such that $\Sigma_{\theta}:=\{\lambda \in \mathbb{C} \backslash\{0\}:|\arg \lambda|<\theta\} \subset \rho(-\mathcal{A})$ and such that for all $\lambda \in \Sigma_{\theta}$ we have

$$
\left\|\lambda(\lambda+\mathcal{A})^{-1}\right\|_{\mathcal{L}(X)} \leq C .
$$

In order to formulate the periodic version of the Da Prato-Grisvard theorem let $\theta \in(0,1)$ and $1 \leq p<\infty$ and recall the space $D_{\mathcal{A}}(\theta, p)$ defined in (1.1). Let $0<T<\infty$ and assume that $f: \mathbb{R} \rightarrow D_{\mathcal{A}}(\theta, p)$ is periodic of period $T$. We recall that the periodic version of (ACP) reads as

$$
\begin{cases}u^{\prime}(t)+\mathcal{A} u(t)=f(t), & t \in \mathbb{R}, \\ u(t)=u(t+T), & t \in \mathbb{R} .\end{cases}
$$

Formally, a candidate for a solution $u$ to (PACP) is given by (1.3). The following lemma shows that, under certain assumptions on $\mathcal{A}$ and $f, u$ is indeed well-defined, continuous, and periodic. 
Lemma 2.1 Let $f: \mathbb{R} \rightarrow D_{\mathcal{A}}(\theta, p)$ be a T-periodic function satisfying $f_{\mid(0, T)} \in L^{p}\left(0, T ; D_{\mathcal{A}}(\theta, p)\right)$ and assume that $0 \in \rho(\mathcal{A})$. Then, the function $u$ defined by (1.3) is well-defined, satisfies $u \in C\left(\mathbb{R} ; D_{\mathcal{A}}(\theta, p)\right)$, and is T-periodic.

Proof Let $k_{0} \in \mathbb{Z}$ be such that $-k_{0} T<t \leq-\left(k_{0}-1\right) T$. Using Hölder's inequality, the periodicity of $f$, and the exponential decay of $\mathrm{e}^{-t \mathcal{A}}$, we obtain

$$
\begin{aligned}
\int_{-\infty}^{t} & \left\|\mathrm{e}^{-(t-s) \mathcal{A}} f(s)\right\|_{D_{\mathcal{A}}(\theta, p)} \mathrm{d} s \\
& =\int_{-k_{0} T}^{t}\left\|\mathrm{e}^{-(t-s) \mathcal{A}} f(s)\right\|_{D_{\mathcal{A}}(\theta, p)} \mathrm{d} s+\sum_{k=k_{0}}^{\infty} \int_{-(k+1) T}^{-k T}\left\|\mathrm{e}^{-(t-s) \mathcal{A}} f(s)\right\|_{D_{\mathcal{A}}(\theta, p)} \mathrm{d} s \\
& \leq C\left(\int_{0}^{t+k_{0} T}\|f(s)\|_{D_{\mathcal{A}}(\theta, p)}^{p} \mathrm{~d} s\right)^{\frac{1}{p}}+C \sum_{k=k_{0}}^{\infty} \mathrm{e}^{-\omega k T} \int_{0}^{T}\left\|\mathrm{e}^{-(T-s) \mathcal{A}} f(s)\right\|_{D_{\mathcal{A}}(\theta, p)} \mathrm{d} s \\
& \leq C\left(1+\sum_{k=k_{0}}^{\infty} \mathrm{e}^{-\omega k T}\right)\left(\int_{0}^{T}\|f(s)\|_{D_{\mathcal{A}}(\theta, p)}^{p} \mathrm{~d} s\right)^{\frac{1}{p}}
\end{aligned}
$$

for some $\omega>0$. It follows that $u$ is well defined. For the continuity of $u$, we write for $h>0$

$$
u(t+h)-u(t)=\int_{t}^{t+h} \mathrm{e}^{-(t+h-s) \mathcal{A}} f(s) \mathrm{d} s+\int_{-\infty}^{t} \mathrm{e}^{-(t-s) \mathcal{A}}\left[\mathrm{e}^{-h \mathcal{A}}-\operatorname{Id}\right] f(s) \mathrm{d} s .
$$

By the boundedness of the semigroup it suffices to consider the second integral. This resembles the expression from the first part of the proof but with $f$ being replaced by $\left[\mathrm{e}^{-h \mathcal{A}}-\mathrm{Id}\right] f$. Thus,

$$
\left\|\int_{-\infty}^{t} \mathrm{e}^{-(t-s) \mathcal{A}}\left[\mathrm{e}^{-h \mathcal{A}}-\operatorname{Id}\right] f(s) \mathrm{d} s\right\|_{D_{\mathcal{A}}(\theta, p)} \leq C\left(\int_{0}^{T}\left\|\left[\mathrm{e}^{-h \mathcal{A}}-\operatorname{Id}\right] f(s)\right\|_{D_{\mathcal{A}}(\theta, p)}^{p} \mathrm{~d} s\right)^{\frac{1}{p}}
$$

and the right-hand side tends to zero as $h \rightarrow 0$ by Lebesgue's theorem. The periodicity of $u$ directly follows by using the transformation $s^{\prime}=s+T$ and the periodicity of $f$.

We now state the periodic version of the Da Prato-Grisvard theorem.

Proposition 2.2 Let $X$ be a Banach space and $-\mathcal{A}$ be the generator of a bounded analytic semigroup on $X$ with $0 \in \rho(\mathcal{A})$. Let $\theta \in(0,1), 1 \leq p<\infty$, and $0<T<\infty$.

Then there exists a constant $C>0$ such that for all T-periodic functions $f: \mathbb{R} \rightarrow D_{\mathcal{A}}(\theta, p)$ with $f_{\mid(0, T)} \in L^{p}\left(0, T ; D_{\mathcal{A}}(\theta, p)\right)$ the function $u$ defined by (1.3) lies in $C\left(\mathbb{R} ; D_{\mathcal{A}}(\theta, p)\right)$, is periodic of period $T$, satisfies $u(t) \in D(\mathcal{A})$ for almost every $t \in \mathbb{R}$, and satisfies

$$
\|\mathcal{A} u\|_{L^{p}\left(0, T ; D_{\mathcal{A}}(\theta, p)\right)} \leq C\|f\|_{L^{p}\left(0, T ; D_{\mathcal{A}}(\theta, p)\right)} \cdot
$$

Proof The continuity and periodicity of $u$ are proven in Lemma 2.1. Let $t \in[0, T)$ and use the transformation $s^{\prime}=s+(k+1) T$ for $k \in \mathbb{N}_{0}$ as well as that $f$ is periodic to write 


$$
u(t)=\int_{0}^{t} \mathrm{e}^{-(t-s) \mathcal{A}} f(s) \mathrm{d} s+\sum_{k=0}^{\infty} \mathrm{e}^{-(t+k T) \mathcal{A}} \int_{0}^{T} \mathrm{e}^{-(T-s) \mathcal{A}} f(s) \mathrm{d} s .
$$

Set $\mathfrak{t}:=\int_{0}^{T} \mathrm{e}^{-(T-s) \mathcal{A}} f(s) \mathrm{d} s$. Since Theorem 1.1 implies

$$
\left.\int_{0}^{t} \mathrm{e}^{-(t-s) \mathcal{A}} f(s) \mathrm{d} s \in D(\mathcal{A}) \quad \text { (a.e. } t \in(0, T)\right)
$$

and

$$
\left\|t \mapsto \mathcal{A} \int_{0}^{t} \mathrm{e}^{-(t-s) \mathcal{A}} f(s) \mathrm{d} s\right\|_{L^{p}\left(0, T ; D_{\mathcal{A}}(\theta, p)\right)} \leq C\|f\|_{L^{p}\left(0, T ; D_{\mathcal{A}}(\theta, p)\right)},
$$

the exponential decay of the semigroup implies that it suffices to prove the estimate

$$
\left\|t \mapsto \mathcal{A} \mathrm{e}^{-t \mathcal{A}} \mathfrak{i t}\right\|_{L^{p}\left(0, T ; D_{\mathcal{A}}(\theta, p)\right)} \leq C\|f\|_{L^{p}\left(0, T ; D_{\mathcal{A}}(\theta, p)\right)} .
$$

Following Lemma 4.14 and Theorem 4.15 of [10], estimate (2.2) holds true provided $\mathfrak{t}$ belongs to the space of traces $\mathcal{T}$ at 0 of functions belonging to the maximal regularity class MR $:=\left\{u \in L^{p}(0, T ; D(\mathcal{A})): u^{\prime}, \mathcal{A} u \in L^{p}\left(0, T ; D_{\mathcal{A}}(\theta, p)\right)\right\}$. Observe that the function $v:[0, T] \rightarrow X, t \mapsto \int_{0}^{t} \mathrm{e}^{-(t-s) \mathcal{A}} f(s) \mathrm{d} s$ belongs to MR and hence so does $v(T-\cdot)$. Consequently, $\mathfrak{t}=v(T) \in \mathcal{T}$ and the proof is complete.

Remark 2.3 We remark that estimate (2.2) can be obtained not only by interpolation methods as explained in the proof above but also by elementary considerations based on Hölder's inequality and Fubini's theorem. For the convenience of the reader, we present here also this elementary proof. To this end, let $\gamma_{1}, \gamma_{2} \in(0,1)$ with $\gamma_{1}+\gamma_{2}=1$ and $1 / p^{\prime}<\gamma_{2}<1-\theta+1 / p^{\prime}$, where $p^{\prime}$ denotes the Hölder conjugate exponent to $p$. Then, the boundedness and the analyticity of the semigroup and Hölder's inequality imply

$$
\begin{aligned}
\left\|\mathcal{A} \mathrm{e}^{-\tau \mathcal{A}} \mathcal{A} \mathrm{e}^{-t \mathcal{A}} \mathfrak{u}\right\|_{X} & \leq C \int_{0}^{T} \frac{1}{(T+\tau+t-s)^{\gamma_{1}}} \frac{1}{(T+\tau+t-s)^{\gamma_{2}}}\left\|\mathcal{A} \mathrm{e}^{-(T+\tau+t-s) / 2 \mathcal{A}} f(s)\right\|_{X} \mathrm{~d} s \\
& \leq C(\tau+t)^{1 / p^{\prime}-\gamma_{2}}\left(\int_{t}^{T+t} \frac{1}{(\tau+s)^{\gamma_{1} p}}\left\|\mathcal{A} \mathrm{e}^{-(\tau+s) / 2 \mathcal{A}} f(T+t-s)\right\|_{X}^{p} \mathrm{~d} s\right)^{\frac{1}{p}} .
\end{aligned}
$$

Next, $t>0$ implies

$$
\left\|\mathcal{A} \mathrm{e}^{-\tau \mathcal{A}} \mathcal{A} \mathrm{e}^{-t \mathcal{A}} \mathfrak{u}\right\|_{X} \leq C \tau^{1 / p^{\prime}-\gamma_{2}}\left(\int_{t}^{T+t} \frac{1}{(\tau+s)^{\gamma_{1} p}}\left\|\mathcal{A} \mathrm{e}^{-(\tau+s) / 2 \mathcal{A}} f(T+t-s)\right\|_{X}^{p} \mathrm{~d} s\right)^{\frac{1}{p}} .
$$

An application of (2.3) and Fubini's theorem yields

$$
\int_{0}^{T}\left\|\mathcal{A} \mathrm{e}^{-\tau \mathcal{A}} \mathcal{A} \mathrm{e}^{-t \mathcal{A}} \mathfrak{u t}\right\|_{X}^{p} \mathrm{~d} t \leq C \tau^{p\left(1 / p^{\prime}-\gamma_{2}\right)} \int_{0}^{2 T} \int_{\max \{0, s-T\}}^{\min \{T, s\}} \frac{1}{(\tau+s)^{\gamma_{1} p}}\left\|\mathcal{A} \mathrm{e}^{-(\tau+s) / 2 \mathcal{A}} f(T+t-s)\right\|_{X}^{p} \mathrm{~d} t \mathrm{~d} s .
$$

as well as

$$
\left\|t \mapsto \mathcal{A} \mathrm{e}^{-\tau \mathcal{A}} \mathcal{A} \mathrm{e}^{-t \mathcal{A}} \mathfrak{i t}\right\|_{L^{p}(0, T ; X)}^{p} \leq C \tau^{p\left(1 / p^{\prime}-\gamma_{2}\right)} \int_{0}^{2 T} \int_{\max \{0, T-s\}}^{T} \frac{1}{(\tau+s)^{\gamma_{1} p}}\left\|\mathcal{A} \mathrm{e}^{-(\tau+s) / 2 \mathcal{A}} f(t)\right\|_{X}^{p} \mathrm{~d} t \mathrm{~d} s .
$$


Another application of Fubini's theorem yields

$$
\int_{0}^{T}\left[\mathcal{A} \mathrm{e}^{-t \mathcal{A}} \mathfrak{u}\right]_{\theta, p}^{p} \mathrm{~d} t \leq C \int_{0}^{\infty} \tau^{\gamma-1} \int_{0}^{2 T} \int_{\max \{0, T-s\}}^{T} \frac{1}{(\tau+s)^{\gamma_{1} p}}\left\|\mathcal{A} \mathrm{e}^{-(\tau+s) / 2 \mathcal{A}} f(t)\right\|_{X}^{p} \mathrm{~d} t \mathrm{~d} s \mathrm{~d} \tau,
$$

where $\gamma=p\left(1+1 / p^{\prime}-\theta-\gamma_{2}\right)$ and

$$
\int_{0}^{T}\left[\mathcal{A} \mathrm{e}^{-t \mathcal{A}} \mathfrak{u}\right]_{\theta, p}^{p} \mathrm{~d} t \leq C \int_{0}^{T} \int_{0}^{\infty} \tau^{\gamma-1} \int_{T+\tau-t}^{2 T+\tau} \frac{1}{s^{\gamma_{1}} p}\left\|\mathcal{A} \mathrm{e}^{-s / 2 \mathcal{A}} f(t)\right\|_{X}^{p} \mathrm{~d} s \mathrm{~d} \tau \mathrm{d} t .
$$

We finally obtain

$$
\int_{0}^{T}\left[\mathcal{A} \mathrm{e}^{-t \mathcal{A}} \mathfrak{i t}\right]_{\theta, p}^{p} \mathrm{~d} t \leq \frac{C}{\gamma} \int_{0}^{T} \int_{T-t}^{\infty} s^{\gamma-\gamma_{1} p}\left\|\mathcal{A} \mathrm{e}^{-s / 2 \mathcal{A}} f(t)\right\|_{X}^{p} \mathrm{~d} s \mathrm{~d} t .
$$

Now, let $\vartheta_{1}, \vartheta_{2}, \vartheta_{3} \in(0,1)$ with $\vartheta_{1}+\vartheta_{2}+\vartheta_{3}=1, \vartheta_{1}<\theta, \vartheta_{2} p^{\prime}<1$ and $\vartheta_{3} p<1$. Since $D_{\mathcal{A}}(\theta, p) \hookrightarrow D\left(\mathcal{A}^{\vartheta_{1}}\right)$ the bounded analyticity of $\mathrm{e}^{-t \mathcal{A}}$, Hölder's inequality, and the above embedding implies

$$
\left\|\mathcal{A} \mathrm{e}^{-t \mathcal{A}} \mathfrak{u}\right\|_{X} \leq C t^{-\vartheta_{3}}\|f\|_{L^{p}\left(0, T ; D_{\mathcal{A}}(\theta, p)\right)}
$$

Consequently,

$$
\int_{0}^{T}\left\|\mathcal{A} \mathrm{e}^{-t \mathcal{A}} \mathfrak{t}\right\|_{X}^{p} \mathrm{~d} t \leq C\|f\|_{L^{p}\left(0, T ; D_{\mathcal{A}}(\theta, p)\right)}^{p}
$$

We conclude this section by showing that, under the assumptions of Proposition 2.2, $u$ defined by (1.3) indeed is the unique strong solution to (PACP).

Proposition 2.4 Under the hypotheses of Proposition 2.2 the function $u$ defined by (1.3) is the unique strong solution to (PACP), i.e., $u$ is the unique periodic function of period $T$ in $C(\mathbb{R} ; X)$ that is for almost every $t \in \mathbb{R}$ differentiable in $t$, satisfies $u(t) \in D(\mathcal{A})$, and $\mathcal{A} u \in L^{p}(0, T ; X)$, and $u$ solves

$$
u^{\prime}(t)+\mathcal{A} u(t)=f(t)
$$

Proof First of all, $u$ is periodic by Lemma 2.1 and since $D_{\mathcal{A}}(\theta, p)$ continuously embeds into $X$ the very same lemma implies $u \in C(\mathbb{R} ; X)$.

Assume first that $f_{\mid(0, T)} \in L^{p}(0, T ; D(\mathcal{A}))$. Then, by a direct calculation, $u$ defined by (1.3) is differentiable, satisfies $u(t) \in D(\mathcal{A})$, and solves

$$
u^{\prime}(t)+\mathcal{A} u(t)=f(t)
$$

for every $t \in \mathbb{R}$. The density of $L^{p}(0, T ; D(\mathcal{A}))$ in $L^{p}\left(0, T ; D_{\mathcal{A}}(\theta, p)\right)$ and the estimate proven in Proposition 2.2 imply that all these properties carry over to all right-hand sides in $L^{p}\left(0, T ; D_{\mathcal{A}}(\theta, p)\right)$ (but only for almost every $t \in \mathbb{R}$ ) by an approximation argument.

For the uniqueness, assume that $v \in C(\mathbb{R} ; X)$ with $v^{\prime}, \mathcal{A} v \in L^{p}(0, T ; X)$ is another periodic function of period $T$ which satisfies the equation for almost every $t \in \mathbb{R}$. Let $w:=u-v$. Then $w$ satisfies 


$$
w^{\prime}(t)=-\mathcal{A} w(t) \quad(\text { a.e. } t \in \mathbb{R}) .
$$

In this case, for $t>0, w$ can be written by means of the semigroup as $w(t)=\mathrm{e}^{-t \mathcal{A}}(u(0)-v(0))$. Now, the exponential decay of the semigroup and the periodicity of $w$ imply that $w$ must be zero for all $t \in \mathbb{R}$.

For $0<T<\infty$, we define the solution space $\mathbb{E}_{\mathcal{A}}^{\text {per }}$ as

$$
\mathbb{E}_{\mathcal{A}}^{\mathrm{per}}:=\left\{u \in \mathrm{W}^{1, p}\left(0, T ; D_{\mathcal{A}}(\theta, p)\right): \mathcal{A} u \in L^{p}\left(0, T ; D_{\mathcal{A}}(\theta, p)\right) \text { and } u(0)=u(T)\right\}
$$

with norm

$$
\|u\|_{\mathbb{E}_{\mathcal{A}}^{\text {per }}}:=\|u\|_{\mathrm{W}^{1, p}\left(0, T ; D_{\mathcal{A}}(\theta, p)\right)}+\|\mathcal{A} u\|_{L^{p}\left(0, T ; D_{\mathcal{A}}(\theta, p)\right)},
$$

which corresponds to the data space

$$
\mathbb{F}_{\mathcal{A}}:=L^{p}\left(0, T ; D_{\mathcal{A}}(\theta, p)\right) .
$$

Combining Propositions 2.2 and 2.4 shows that for each periodic $f$ with period $T$ and $f_{\mid(0, T)} \in L^{p}\left(0, T ; D_{\mathcal{A}}(\theta, p)\right)$ also $u_{\mid(0, T)}^{\prime} \in L^{p}\left(0, T ; D_{\mathcal{A}}(\theta, p)\right)$. The same is true for $u$ since $0 \in \rho(\mathcal{A})$. Summarizing, there exists a constant $C>0$ such that

$$
\|u\|_{\mathbb{E}_{\mathcal{A}}^{\text {per }}} \leq C\|f\|_{L^{p}\left(0, T ; D_{\mathcal{A}}(\theta, p)\right)} .
$$

\section{Time-periodic solutions for semilinear equations}

In this section, we use the periodic version of the Da Prato-Grisvard theorem to construct time-periodic solutions to semilinear parabolic equations by employing Banach's fixed point theorem.

\subsection{An existence theorem for general types of nonlinearities}

Let $-\mathcal{A}$ be the generator of a bounded analytic semigroup $\mathrm{e}^{-t \mathcal{A}}$ on a Banach space $X$ with the domain $D(\mathcal{A})$ and $0 \in \rho(\mathcal{A})$. For $T>0, \theta \in(0,1)$, and $1 \leq p<\infty$ let $f: \mathbb{R} \rightarrow D_{\mathcal{A}}(\theta, p)$ be periodic of period $T$ with $f_{\mid(0, T)} \in L^{p}\left(0, T ; D_{\mathcal{A}}(\theta, p)\right)$. We are aiming for the strong solvability of

$$
\begin{cases}u^{\prime}(t)+\mathcal{A} u(t)=F[u](t)+f(t), & t \in \mathbb{R}, \\ u(t)=u(t+T), & t \in \mathbb{R}\end{cases}
$$

under some smallness assumptions on $f$. The solution $u$ will be constructed in the space of maximal regularity $\mathbb{E}_{\mathcal{A}}^{\text {per }}$ defined in (2.5). Recall the corresponding data space

$$
\mathbb{F}_{\mathcal{A}}=L^{p}\left(0, T ; D_{\mathcal{A}}(\theta, p)\right)
$$

and let $\mathbb{B}_{\rho}:=\mathbb{B}^{\mathbb{E}_{\mathcal{A}}^{\text {per }}}(0, \rho)$ denote the ball in $\mathbb{E}_{\mathcal{A}}^{\text {per }}$ centered at 0 and with radius $\rho>0$. For the nonlinear term $F$, we make the following standard assumption. 
Assumption $\mathbf{N}$ There exists $R>0$ such that the nonlinear term $F$ is a mapping from $\mathbb{B}_{R}$ into $\mathbb{F}_{\mathcal{A}}$ and satisfies

$$
F \in C^{1}\left(\mathbb{B}_{R} ; \mathbb{F}_{\mathcal{A}}\right), \quad F(0)=0, \quad \text { and } \quad \mathrm{DF}(0)=0,
$$

where DF : $\mathbb{B}_{R} \rightarrow \mathcal{L}\left(\mathbb{E}_{\mathcal{A}}^{\text {per }}, \mathbb{F}_{\mathcal{A}}\right)$ denotes the Fréchet derivative.

The following theorem proves existence and uniqueness of solutions to (NACP) in the class $\mathbb{E}_{\mathcal{A}}^{\text {per }}$ for small forcings $f$.

Theorem 3.1 Let $T>0,0<\theta<1,1 \leq p<\infty$, and $F$ and $R>0$ subject to Assumption $\mathrm{N}$. Then there is a constant $r \leq R$ and $c=c(T, \theta, p, r)>0$ such that if $f: \mathbb{R} \rightarrow D_{\mathcal{A}}(\theta, p)$ is T-periodic with $\|f\|_{\mathbb{F}_{A}} \leq c$, then there exists a unique solution $u: \mathbb{R} \rightarrow D_{\mathcal{A}}(\theta, p)$ of (NACP) with the same period $T$ and $u_{\mid(0, T)} \in \mathbb{B}_{r}$.

Proof Let $S: \mathbb{B}_{R} \rightarrow \mathbb{E}_{\mathcal{A}}^{\text {per }}, v \mapsto u_{v}$ be the solution operator of the linear equation

$$
u_{v}^{\prime}(t)+\mathcal{A} u_{v}(t)=F[v(t)]+f(t) \quad \text { in }(0, T)
$$

with $u_{v}(0)=u_{v}(T)$. This is well-defined since $F[v] \in \mathbb{F}_{\mathcal{A}}$ by Assumption $\mathrm{N}$, so that, by Proposition 2.4 and the remarks at the end of Sect. $2, u_{v}$ uniquely exists and lies in $\mathbb{E}_{\mathcal{A}}^{\text {per }}$.

We prove that this solution operator is a contraction on $\mathbb{B}_{r}$ for some $r \leq R$. Let $M>0$ denote the infimum of all constants $C$ satisfying (2.6). Choose $r>0$ small enough such that

$$
\sup _{w \in \mathbb{B}_{r}}\|\mathrm{DF}[w]\|_{\mathcal{L}\left(\mathbb{E}_{\mathcal{A}}^{\text {per }}, \mathbb{F}_{\mathcal{A}}\right)} \leq \frac{1}{2 M},
$$

which is possible by Assumption N. By virtue of (2.6) as well as the mean value theorem, estimate for any $v \in \mathbb{B}_{r}$ and $f$ satisfying $\|f\|_{\mathbb{F}_{\mathcal{A}}} \leq r /(2 M)=: c$,

$$
\|S(v)\|_{\mathbb{E}_{\mathcal{A}}^{\text {per }}} \leq M\left(\|F[v]\|_{\mathbb{F}_{\mathcal{A}}}+\|f\|_{\mathbb{F}_{\mathcal{A}}}\right) \leq M\left(\sup _{w \in \mathbb{B}_{r}}\|\mathrm{DF}[w]\|_{\mathcal{L}\left(\mathbb{E}_{\mathcal{A}}^{\text {per }}, \mathbb{F}_{\mathcal{A}}\right)}\|v\|_{\mathbb{E}_{\mathcal{A}}^{\text {per }}}+\|f\|_{\mathbb{F}_{\mathcal{A}}}\right) \leq r .
$$

So $S\left(\mathbb{B}_{r}\right) \subset \mathbb{B}_{r}$. Similarly, for any $v_{1}, v_{2} \in \mathbb{B}_{r}$,

$$
\left\|S\left(v_{1}\right)-S\left(v_{2}\right)\right\|_{\mathbb{E}_{\mathcal{A}}^{\text {per }}} \leq M \sup _{w \in \mathbb{B}_{r}}\|\mathrm{DF}[w]\|_{\mathcal{L}\left(\mathbb{E}_{\mathcal{A}}^{\mathrm{per}}, \mathbb{F}_{\mathcal{A}}\right)}\left\|v_{1}-v_{2}\right\|_{\mathbb{E}_{\mathcal{A}}^{\mathrm{per}}} \leq \frac{1}{2}\left\|v_{1}-v_{2}\right\|_{\mathbb{E}_{\mathcal{A}}^{\text {per }}} .
$$

Consequently, the solution operator $S$ is a contraction on $\mathbb{B}_{r}$ and the contraction mapping theorem is applicable. The solution to (NACP) is defined as follows. Let $u$ be the unique fixed point of $S$. Since $S u=u, u$ satisfies $u(0)=u(T)$ and thus can be extended periodically to the whole real line. This function solves (NACP).

\section{Periodic solutions to the bidomain equations}

Let the space dimension $n \geq 2$ be fixed and let $\Omega \subset \mathbb{R}^{n}$ denote a bounded domain with boundary $\partial \Omega$ of class $C^{2}$. For the conductivity matrices $\sigma_{\mathrm{i}}$ and $\sigma_{\mathrm{e}}$, we make the following assumptions. 
Assumption $\mathbf{E}$ The conductivity matrices $\sigma_{\mathrm{i}}, \sigma_{\mathrm{e}}: \bar{\Omega} \rightarrow \mathbb{R}^{n \times n}$ are symmetric matrices and are functions of class $C^{1}(\bar{\Omega})$. Ellipticity is imposed by means of the following condition: there exist constants $\underline{\sigma}, \bar{\sigma}$ with $0<\underline{\sigma}<\bar{\sigma}$ such that

$$
\underline{\sigma}|\xi|^{2} \leq\left\langle\sigma_{\mathrm{i}}(x) \xi, \xi\right\rangle \leq \bar{\sigma}|\xi|^{2} \quad \text { and } \quad \underline{\sigma}|\xi|^{2} \leq\left\langle\sigma_{\mathrm{e}}(x) \xi, \xi\right\rangle \leq \bar{\sigma}|\xi|^{2}
$$

for all $x \in \bar{\Omega}$ and all $\xi \in \mathbb{R}^{n}$. Moreover, it is assumed that

$$
\begin{array}{llll}
\sigma_{\mathrm{i}} \nabla u_{\mathrm{i}} \cdot v=0 & \Leftrightarrow & \nabla u_{\mathrm{i}} \cdot v=0 & \text { on } \partial \Omega, \\
\sigma_{\mathrm{e}} \nabla u_{\mathrm{e}} \cdot v=0 & \Leftrightarrow & \nabla u_{\mathrm{e}} \cdot v=0 & \text { on } \partial \Omega .
\end{array}
$$

It is known that (4.2) is a biological reasonable assumption.

Next, we define the bidomain operator in the $L^{q}$-setting for $1<q<\infty$. To this end, let $L_{a v}^{q}(\Omega):=\left\{u \in L^{q}(\Omega): \int_{\Omega} u \mathrm{~d} x=0\right\}$ and let $P_{a v}$ be the canonical projection from $L^{q}(\Omega)$ to $L_{a v}^{q}(\Omega)$, i.e., $P_{a v} u:=u-\frac{1}{|\Omega|} \int_{\Omega} u \mathrm{~d} x$. We then introduce the operators $A_{\mathrm{i}}$ and $A_{\mathrm{e}}$ by

$$
\begin{aligned}
A_{i, e} u & :=-\nabla \cdot\left(\sigma_{i, e} \nabla u\right), \\
D\left(A_{i, e}\right) & :=\left\{u \in \mathrm{W}^{2, q}(\Omega) \cap L_{a v}^{q}(\Omega): \sigma_{i, e} \nabla u \cdot v=0 \text { a.e. on } \partial \Omega\right\} \subset L_{a v}^{q}(\Omega),
\end{aligned}
$$

where $A_{i, e}$ and $\sigma_{i, e}$ indicates that either $A_{\mathrm{i}}$ and $\sigma_{\mathrm{i}}$ or $A_{\mathrm{e}}$ and $\sigma_{\mathrm{e}}$ are considered. Due to condition (4.2), we obtain $D\left(A_{\mathrm{i}}\right)=D\left(A_{\mathrm{e}}\right)$, and thus, it is possible to define the sum $A_{\mathrm{i}}+A_{\mathrm{e}}$ of $A_{\mathrm{i}}$ and $A_{\mathrm{e}}$ with the domain $D\left(A_{\mathrm{i}}\right)=D\left(A_{\mathrm{e}}\right)$. Note that the inverse operator $\left(A_{\mathrm{i}}+A_{\mathrm{e}}\right)^{-1}$ on $L_{a v}^{q}(\Omega)$ is a bounded linear operator.

Following [15], we define the bidomain operator as follows. Let $\sigma_{\mathrm{i}}$ and $\sigma_{\mathrm{e}}$ satisfy Assumption E. Then the bidomain operator $A$ is defined as

$$
A=A_{\mathrm{i}}\left(A_{\mathrm{i}}+A_{\mathrm{e}}\right)^{-1} A_{\mathrm{e}} P_{a v}
$$

with domain

$$
D(A):=\left\{u \in \mathrm{W}^{2, q}(\Omega): \nabla u \cdot v=0 \text { a.e. on } \partial \Omega\right\} .
$$

The following result for $A$ was proven by Giga and Kajiwara in [15] using a contradiction argument. For a direct proof and the $\mathrm{H}^{\infty}$-calculus property of $A$, see [19].

Proposition 4.1 [15, Theorem 4.7, Theorem 4.9], [19] Let $1<q<\infty, \Omega$ be a bounded $C^{2}$-domain and let $\sigma_{\mathrm{i}}$ and $\sigma_{\mathrm{e}}$ satisfy Assumption E. Then $-A$ generates a bounded analytic semigroup $\mathrm{e}^{-t A}$ on $L^{q}(\Omega)$.

Under the assumption of the conservation of currents, i.e.,

$$
\int_{\Omega}\left(I_{\mathrm{i}}(t)+I_{\mathrm{e}}(t)\right) \mathrm{d} x=0, \quad t \geq 0
$$

and assuming moreover $\int_{\Omega} u_{\mathrm{e}} \mathrm{d} x=0$, the bidomain equations (BDE) may be equivalently rewritten as an evolution equation $[6,15]$ of the form

$$
\begin{cases}\partial_{t} u+A u+F(u, w)=I, & \text { in }(0, \infty), \\ \partial_{t} w+G(u, w)=0, & \text { in }(0, \infty), \\ u(0)=u_{0}, & \\ w(0)=w_{0}, & \end{cases}
$$


where

$$
I:=I_{\mathrm{i}}-A_{\mathrm{i}}\left(A_{\mathrm{i}}+A_{\mathrm{e}}\right)^{-1}\left(I_{\mathrm{i}}+I_{\mathrm{e}}\right)
$$

is the modified source term. The functions $u_{\mathrm{e}}$ and $u_{\mathrm{i}}$ can be recovered from $u$ by virtue of the following relations

$$
\begin{aligned}
& u_{\mathrm{e}}=\left(A_{\mathrm{i}}+A_{\mathrm{e}}\right)^{-1}\left\{\left(I_{\mathrm{i}}+I_{\mathrm{e}}\right)-A_{\mathrm{i}} P_{a v} u\right\}, \\
& u_{\mathrm{i}}=u+u_{\mathrm{e}} .
\end{aligned}
$$

For the situation of the bidomain operator $A$, the solution space for $u$ reads as

$$
\mathbb{E}_{A}^{\mathrm{per}}=\left\{u \in \mathrm{W}^{1, p}\left(0, T ; D_{A}(\theta, p)\right): A u \in L^{p}\left(0, T ; D_{A}(\theta, p)\right) \text { and } u(0)=u(T)\right\} .
$$

The solution space for the gating variable $w$ is defined as

$$
\mathbb{E}_{w}^{\text {per }}:=\left\{w \in \mathrm{W}^{1, p}\left(0, T ; D_{A}(\theta, p)\right): w(0)=w(T)\right\} .
$$

Then, the solution space for the periodic bidomain system is defined as the product space

$$
\mathbb{E}:=\mathbb{E}_{A}^{\text {per }} \times \mathbb{E}_{w}^{\text {per }}
$$

Finally, for a Banach space $X$ we denote by $\mathbb{B}^{X}\left(u^{*}, R\right)$ the closed ball in $X$ with center $u^{*} \in X$ and radius $R>0$, i.e.,

$$
\mathbb{B}^{X}\left(u^{*}, R\right):=\left\{u \in X:\left\|u-u^{*}\right\|_{X} \leq R\right\} .
$$

We now state our main results concerning the existence and uniqueness of strong $T$-periodic solutions to the bidomain equations subject to various models of the ionic transport. Notice that the respective models treated here are slightly more general as described in the introduction, as an additional parameter $\varepsilon>0$ is introduced, that incorporates the phenomenon of fast and slow diffusion.

Additionally to Assumption $\mathrm{E}$ on the conductivity matrices of the bidomain operator $A$, we require the following regularity and periodicity conditions on the forcing term $I$.

Assumption $\mathbf{P}$ Let $2 \theta \in(0,1), 1 \leq p<\infty$, and $1<q<\infty$ satisfy $2 \theta>n / q$ or, if $p=1$ let $2 \theta \geq n / q$. Assume $I: \mathbb{R} \rightarrow D_{A}(\theta, p)$ is a $T$-periodic function satisfying $I_{\mid(0, T)} \in \mathbb{F}_{A}$ for some $\theta \in(0,1 / 2)$ and $T>0$.

Remark 4.2 If $\Omega$ has a $C^{4}$-boundary and if the conductivity matrices $\sigma_{\mathrm{i}}$ and $\sigma_{\mathrm{e}}$ lie in $\mathrm{W}^{3, \infty}\left(\Omega ; \mathbb{R}^{n \times n}\right)$, then Assumption $\mathrm{P}$ is satisfied by virtue of (4.5) if $I_{\mathrm{i}}, I_{\mathrm{e}}: \mathbb{R} \rightarrow D_{A}(\theta, p)$ are $T$-periodic functions satisfying $I_{\left.i\right|_{(O T)}}$ and $I_{\left.e\right|_{(O T)}} \in \mathbb{F}_{A}$. Indeed, this follows by real interpolation since $A_{\mathrm{i}}\left(A_{\mathrm{i}}+A_{\mathrm{e}}\right)^{-1}$ is bounded on $L_{a v}^{q}(\Omega)$ and from $D(A)$ into $\mathrm{W}^{2, q}(\Omega) \cap L_{a v}^{q}(\Omega)$.

We start with the most classical model due to FitzHugh and Nagumo.

\subsection{The periodic bidomain FitzHugh-Nagumo model}

For $T>0,0<a<1$, and $b, c, \varepsilon>0$, the periodic bidomain FitzHugh-Nagumo equations are given by 


$$
\begin{cases}\partial_{t} u+\varepsilon A u=I-\frac{1}{\varepsilon}\left[u^{3}-(a+1) u^{2}+a u+w\right] & \text { in } \mathbb{R} \times \Omega, \\ \partial_{t} w=c u-b w & \text { in } \mathbb{R} \times \Omega, \\ u(t)=u(t+T) & \text { in } \mathbb{R} \times \Omega, \\ w(t)=w(t+T) & \text { in } \mathbb{R} \times \Omega .\end{cases}
$$

This system has three equilibrium points, the trivial one $\left(u_{1}, w_{1}\right)=(0,0)$ and two others given by $\left(u_{2}, w_{2}\right)$ and $\left(u_{3}, w_{3}\right)$, where

$$
\begin{array}{ll}
u_{2}=\frac{1}{2}(a+1-d), & w_{2}=\frac{c}{2 b}(a+1-d), \\
u_{3}=\frac{1}{2}(a+1+d), & w_{3}=\frac{c}{2 b}(a+1+d),
\end{array}
$$

and $d=\sqrt{(a+1)^{2}-4\left(a+\frac{c}{b}\right)}$. We assume that the following stability condition $\left(\mathrm{S}_{F N}\right)$ on the coefficients is satisfied:

$$
c<b\left(\frac{(a-1)^{2}}{4}-a\right) \text { and } u_{3}>\frac{1}{3}\left(a+1+\sqrt{(a+1)^{2}-3 a}\right) .
$$

Our result on strong periodic solutions to the bidomain FitzHugh-Nagumo equations reads then as follows.

Theorem 4.3 Let $\Omega \subset \mathbb{R}^{n}, n \geq 2$, be a bounded $C^{2}$-domain and suppose that Assumptions $\mathrm{E}$ and $\mathrm{P}$ hold true.

(a) Then there exist constants $R>0$ and $C(R)>0$ such that if $\|I\|_{\mathbb{F}_{A}}<C(R)$, Eq. (4.6) admits a unique T-periodic strong solution $(u, w)$ with $(u, w)_{\mid(0, T)} \in \mathbb{B}^{\mathbb{E}}((0,0), R)$.

(b) If condition $\left(\mathrm{S}_{\mathrm{FN}}\right)$ is satisfied, then there exist constants $R>0$ and $C(R)>0$ such that if $\|I\|_{\mathbb{F}_{A}}<C(R), E q$. (4.6) admits a unique T-periodic strong solution $(u, w)$ with $(u, w)_{\mid(0, T)} \in \mathbb{B}^{\mathbb{E}}\left(\left(u_{3}, w_{3}\right), R\right)$.

\subsection{The periodic bidomain Aliev-Panfilov model}

For $T>0,0<a<1$, and $d, k, \varepsilon>0$, the periodic bidomain Aliev-Panfilov equations are given by

$$
\begin{cases}\partial_{t} u+\varepsilon A u=I-\frac{1}{\varepsilon}\left[k u^{3}-k(a+1) u^{2}+k a u+u w\right] & \text { in } \mathbb{R} \times \Omega, \\ \partial_{t} w=-(k u(u-1-a)+d w) & \text { in } \mathbb{R} \times \Omega, \\ u(t)=u(t+T) & \text { in } \mathbb{R} \times \Omega, \\ w(t)=w(t+T) & \text { in } \mathbb{R} \times \Omega .\end{cases}
$$

This system has only one stable equilibrium point, namely the trivial solution $\left(u_{1}, w_{1}\right)=(0,0)$. Our theorem on the existence and uniqueness of strong, periodic solutions to the periodic bidomain Aliev-Panfilov equations reads as follows.

Theorem 4.4 Let $\Omega \subset \mathbb{R}^{n}, n \geq 2$, be a bounded $C^{2}$-domain and suppose that Assumptions $\mathrm{E}$ and $\mathrm{P}$ hold true. Then, there exist constants $R>0$ and $C(R)>0$ such that 
if $\|I\|_{\mathbb{F}_{A}}<C(R), \quad E q$. (4.8) admits a unique T-periodic strong solution $(u, w)$ with $(u, w)_{\mid(0, T)} \in \mathbb{B}^{\mathbb{E}}((0,0), R)$.

\subsection{The periodic bidomain Rogers-McCulloch model}

For $T>0,0<a<1$, and $b, c, d, \varepsilon>0$, the periodic bidomain Rogers-McCulloch equations are given by

$$
\begin{cases}\partial_{t} u+\varepsilon A u=I-\frac{1}{\varepsilon}\left[b u^{3}-b(a+1) u^{2}+b a u+u w\right] & \text { in } \mathbb{R} \times \Omega, \\ \partial_{t} w=c u-d w & \text { in } \mathbb{R} \times \Omega, \\ u(t)=u(t+T) & \text { in } \mathbb{R} \times \Omega, \\ w(t)=w(t+T) & \text { in } \mathbb{R} \times \Omega .\end{cases}
$$

This system has three equilibrium points, the trivial one $\left(u_{1}, w_{1}\right)=(0,0)$ and two others given by $\left(u_{2}, w_{2}\right)$ and $\left(u_{3}, w_{3}\right)$, where

$$
\begin{aligned}
& u_{2}=\frac{1}{2}\left(a+1-\frac{c}{b d}-e\right), \quad w_{2}=\frac{c}{2 d}\left(a+1-\frac{c}{b d}-e\right), \\
& u_{3}=\frac{1}{2}\left(a+1-\frac{c}{b d}+e\right), \quad w_{3}=\frac{c}{2 d}\left(a+1-\frac{c}{b d}+e\right),
\end{aligned}
$$

and $e=\sqrt{\left(a+1-\frac{c}{b d}\right)^{2}-4 a}$. We assume that the following stability condition $\left(\mathrm{S}_{\mathrm{RM}}\right)$ on the coefficients is satisfied:

$$
\sqrt{\left(a+1-\frac{c}{b d}\right)^{2}-4 a}-\frac{c}{b d}>0 .
$$

Our theorem on the existence and uniqueness of strong periodic solutions to the periodic bidomain Rogers-McCulloch equations reads as follows.

Theorem 4.5 Let $\Omega \subset \mathbb{R}^{n}, n \geq 2$, be a bounded $C^{2}$-domain and suppose that Assumptions $\mathrm{E}$ and $\mathrm{P}$ hold true.

(a) Then there exist constants $R>0$ and $C(R)>0$ such that if $\|I\|_{\mathbb{F}_{A}}<C(R)$, Eq. (4.9) admits a unique T-periodic strong solution $(u, w)$ with $(u, w)_{\mid(0, T)} \in \mathbb{B}^{\mathbb{E}}((0,0), R)$.

(b) If condition $\left(\mathrm{S}_{\mathrm{RM}}\right)$ is satisfied, then there exist constants $R>0$ and $C(R)>0$ such that if $\|I\|_{\mathbb{F}_{A}}<C(R), E q$. (4.9) admits a unique T-periodic strong solution $(u, w)$ with $(u, w)_{\mid(0, T)} \in \mathbb{B}^{\mathbb{E}}\left(\left(u_{3}, w_{3}\right), R\right)$.

\subsection{The periodic bidomain Allen-Cahn equation}

For $T>0$, the periodic bidomain Allen-Cahn equation is given by

$$
\begin{cases}\partial_{t} u+A u=I+u-u^{3} & \text { in } \mathbb{R} \times \Omega, \\ u(t)=u(t+T) & \text { in } \mathbb{R} \times \Omega .\end{cases}
$$


This system has three equilibrium points, $u_{1}=-1, u_{2}=0$, and $u_{3}=1$ and our theorem on the existence and uniqueness of strong, periodic solutions to the periodic bidomain Allen-Cahn equation reads as follows.

Theorem 4.6 Let $\Omega \subset \mathbb{R}^{n}, n \geq 2$, be a bounded $C^{2}$-domain and suppose that Assumptions $\mathrm{E}$ and $\mathrm{P}$ hold true.

(a) Then, there exist constants $R>0$ and $C(R)>0$ such that if $\|I\|_{\mathbb{F}_{A}}<C(R) E q$. (4.12)

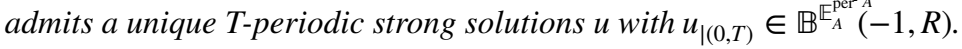

(b) Then, there exist constants $R>0$ and $C(R)>0$ such that if $\|I\|_{\mathbb{F}_{A}}<C(R) E q$. (4.12)

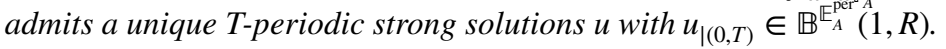

\section{Proofs of the theorems related to the bidomain equations}

The proofs of the results presented in Sect. 4 will be subdivided in several subsections. We start with a result on the linear part of the bidomain systems. The linear part of the bidomain system will be represented as an operator matrix and it will be eminent that the negative of this operator matrix generates a bounded analytic semigroup.

\subsection{Linear theory}

Let $-B$ be the generator of a bounded analytic semigroup on a Banach space $X_{1}$ with $0 \in \rho(B), 1 \leq p<\infty$, and $\theta \in(0,1)$. Let $X_{2}=D_{B}(\theta, p)$ and define for $d>0$ and $b, c \geq 0$ the operator $\mathcal{A}: X:=X_{1} \times X_{2} \rightarrow X$ with domain $D(\mathcal{A}):=D(B) \times X_{2}$ by

$$
\mathcal{A}:=\left(\begin{array}{cc}
B & b \\
-c & d
\end{array}\right)
$$

Lemma 5.1 Then $-\mathcal{A}$ generates a bounded analytic semigroup on $X$ with $0 \in \rho(\mathcal{A})$.

Proof Let $\Sigma_{\omega}, \omega \in(\pi / 2, \pi]$, be a sector that satisfies $\rho(-B) \subset \Sigma_{\omega}$ with

$$
\left\|\lambda(\lambda+B)^{-1}\right\|_{\mathcal{L}\left(X_{1}\right)} \leq C \quad\left(\lambda \in \Sigma_{\omega}\right) .
$$

First note that $0 \in \rho(\mathcal{A})$; its inverse being

$$
\mathcal{A}^{-1}=\left(\begin{array}{cc}
d & -b \\
c & B
\end{array}\right)(b c+d B)^{-1} .
$$

Note that the choice $X_{2}=D_{B}(\theta, p)$ is used here as $\mathcal{A}^{-1}$ is only an operator from $X_{1} \times X_{2}$ onto $D(B) \times X_{2}$ if $D(B) \subset X_{2} \subset X_{1}$ and if $B(b c+d B)^{-1}$ maps $X_{2}$ into $X_{2}$. By the definition of $D_{B}(\theta, p)$ in (1.1) this latter is satisfied.

For the resolvent problem let $\lambda \in \Sigma_{\beta}, \beta \in(\pi / 2, \omega)$ to be chosen. Then, 


$$
(\lambda+\mathcal{A})^{-1}=(\lambda+d)^{-1}\left(\begin{array}{cc}
\lambda+d & -b \\
c & \lambda+B
\end{array}\right)\left(\lambda+\frac{b c}{\lambda+d}+B\right)^{-1}
$$

whenever $\lambda+\frac{b c}{\lambda+d} \in \rho(-B)$. To determine the angle $\beta$ for which $\lambda+\frac{b c}{\lambda+d} \in \rho(-B)$ distinguish between the cases $|\lambda|<M$ and $|\lambda| \geq M$ for some suitable constant $M>0$. Notice that only the case $b, c>0$ is of interest. Let $C_{\omega}>0$ be a constant depending solely on $\omega$ such that $|\lambda+d| \geq C_{\omega}(|\lambda|+d)$. Choose $M$ such that $|\lambda| \geq M$ if and only if

$$
C_{\omega} \sin (\omega-\beta)\left[|\lambda|^{2}+d|\lambda|\right] \geq 2 b c .
$$

This implies

$$
\left|\frac{b c}{\lambda+d}\right| \leq \frac{b c}{C_{\omega}(|\lambda|+d)} \leq \frac{|\lambda| \sin (\omega-\beta)}{2}
$$

and thus that $\lambda+\frac{b c}{\lambda+d} \in \Sigma_{\omega}$. Moreover,

$$
\left|\lambda+\frac{b c}{d+\lambda}\right| \geq|\lambda|\left(1-\frac{\sin (\omega-\beta)}{2}\right) .
$$

Next, choose $\beta$ that close to $\pi / 2$ such that

$$
M \sin (\beta-\pi / 2) \leq \frac{b c d}{b c+(d+M)^{2}} .
$$

Notice that $M$ itself depends on $\beta$, however, it depends only uniformly on its distance to $\omega$ by (5.1). In the case $|\lambda|<M$ the validity of (5.3) together with trigonometric considerations implies that $\operatorname{Re}\left(\lambda+\frac{b c}{d+\lambda}\right) \geq 0$ proving that under conditions (5.1) and (5.3) we have $\lambda+\frac{b c}{d+\lambda} \in \Sigma_{\omega}$ whenever $\lambda \stackrel{d+\lambda}{\in} \Sigma_{\beta}$. We conclude that $\lambda \in \rho(-\mathcal{A})$. To obtain the resolvent estimate, we calculate

$$
\begin{gathered}
\left\|\lambda(\lambda+\mathcal{A})^{-1}\right\|_{\mathcal{L}(X)} \leq\left\|\lambda\left(\lambda+\frac{b c}{\lambda+d}+B\right)^{-1}\right\|_{\mathcal{L}\left(X_{1}\right)}+\left|\frac{\lambda b}{\lambda+d}\right|\left\|\left(\lambda+\frac{b c}{\lambda+d}+B\right)^{-1}\right\|_{\mathcal{L}\left(X_{2}, X_{1}\right)} \\
+\left|\frac{\lambda c}{\lambda+d}\right|\left\|\left(\lambda+\frac{b c}{\lambda+d}+B\right)^{-1}\right\|_{\mathcal{L}\left(X_{1}, X_{2}\right)}+\left|\frac{\lambda}{\lambda+d}\right|\left\|(\lambda+B)\left(\lambda+\frac{b c}{\lambda+d}+B\right)^{-1}\right\|_{\mathcal{L}\left(X_{2}\right)} .
\end{gathered}
$$

The first term on the right-hand side is directly handled by the resolvent estimate of $B$. The second is treated by this resolvent estimate as well and by noting that $X_{2} \subset X_{1}$. The fourth term is estimated by using that the definition of $X_{2}$ in (1.1) implies resolvent estimates in $X_{2}$ [the resolvent commutes with the semigroup appearing in (1.1)]. For the third term, the estimate follows from the invertibility of $B$ and the interpolation inequality $\|x\|_{X_{2}} \leq C\|x\|_{X_{1}}^{1-\theta}\|B x\|_{X_{1}}^{\theta}$. Altogether, this yields

$$
\begin{aligned}
\left\|\lambda(\lambda+\mathcal{A})^{-1}\right\|_{\mathcal{L}(X)} \leq & C\left(|\lambda|+\left|\frac{\lambda b}{\lambda+d}\right|+\left|\frac{\lambda c}{\lambda+d}\right|\left|\lambda+\frac{b c}{\lambda+d}\right|^{\theta}+\left|\frac{\lambda^{2}}{\lambda+d}\right|\right)\left|\lambda+\frac{b c}{\lambda+d}\right|^{-1} \\
& +C\left|\frac{\lambda}{\lambda+d}\right| .
\end{aligned}
$$

The resolvent estimate for $|\lambda| \geq M$ follows by means of the uniform boundedness of the term $|\lambda /(\lambda+d)|$ and by (5.2).

For $|\lambda|<M$ the function $\lambda \mapsto \lambda(\lambda+\mathcal{A})^{-1}$ is continuous on $\overline{\Sigma_{\beta}} \cap \overline{B(0, M)}$ since $0 \in \rho(\mathcal{A})$. This implies the resolvent estimate also for small $\lambda$. 


\subsection{The bidomain equations as semilinear evolution equations}

A short glimpse toward the models presented in Sects. 4.1-4.4 reveals that one of the following situations occurs:

- The bidomain operator $A$ appears only in the first but not in the second equation of the bidomain models and the nonlinearity depends linearly on the gating variable $w$. (Sects. 4.1-4.3)

- The ODE and the gating variable $w$ are omitted. (Sect. 4.4)

As a consequence, in the first situation the operator associated with the linearization of the bidomain models can be written as an operator matrix whose first component of the domain embeds into a $\mathrm{W}^{2, q}$-space. Since the dynamics of the gating variable is described only by an ODE, there appears no smoothing in the spatial variables of $w$. However, as we aim to employ Theorem 3.1 and as the nonlinearity of the first equation depends linearly on $w$, at least in the models of Aliev-Panfilov and Rogers-McCulloch, $w$ must be contained in $D_{A}(\theta, p)$. Otherwise, one cannot view the nonlinearity as a suitable right-hand side as it is done in Sect. 3.1. Hence, we choose $D_{A}(\theta, p)$ as the ground space for the gating variable.

To describe this situation in our setup, assume in the following, that $-\mathcal{A}$ is the generator of a bounded analytic semigroup on a Banach space $X=X_{1} \times X_{2}$, with domain $D(\mathcal{A})=D\left(A_{1}\right) \times D\left(A_{2}\right)$, and $0 \in \rho(\mathcal{A})$. We further set for some $1<q<\infty, 1 \leq p<\infty$, and $\theta \in(0,1)$

$$
X_{1}=L^{q}(\Omega), \quad D\left(A_{1}\right)=D(A), \quad \text { and } \quad X_{2}=D\left(A_{2}\right)=D_{A}(\theta, p) .
$$

Furthermore, define two types of nonlinearities as follows: For $a_{1}, a_{2}, a_{3}, a_{4} \in \mathbb{R}$ let

$$
F_{1}\left[u_{1}, u_{2}\right]:=\left(\begin{array}{c}
a_{1} u_{1}^{2}+a_{2} u_{1}^{3}+a_{3} u_{1} u_{2} \\
a_{4} u_{1}^{2}
\end{array}\right)
$$

and for $b_{1}, b_{2} \in \mathbb{R}$ let

$$
F_{2}\left[u_{1}\right]:=b_{1} u_{1}^{2}+b_{2} u_{1}^{3} .
$$

Here, $F_{1}$ will be a prototype of the nonlinearities considered in Sects. $4.1-4.3$ and $F_{2}$ for the one considered in Sect. 4.4. For the moment, the condition $0 \in \rho(\mathcal{A})$ seems inappropriate as $0 \notin \rho(A)$. However, we will linearize the bidomain equations around suitable stable stationary solutions and in this situation $0 \in \rho(\mathcal{A})$ will be achieved.

In the following, we concentrate only on $F_{1}$, since the results for $F_{2}$ may be proved in a similar way. To derive conditions on $p, q$, and $\theta$ ensuring that $F_{1}$ satisfies Assumption $\mathrm{N}$, the following lemma is essential.

Lemma 5.2 Let $\Omega \subset \mathbb{R}^{n}$ be a bounded $C^{2}$-domain. Let $s>0$ and $p, q \in[1, \infty)$ with $s>n / q$ or, in the case $p=1$, let $s \geq n / q$. Then $\mathrm{B}_{q, p}^{s}(\Omega)$ is a Banach algebra.

Proof If $\Omega=\mathbb{R}^{n}$ and $s>n / q$ this readily follows by Sobolev's embedding combined with [5, Cor. 2.86]. If $s=n / q$ and $p=1$, then [5, Cor. 2.86] has to be combined with [5, Prop. 2.39]. Finally, the algebra property on domains is directly transferred from the whole space since bounded $C^{2}$-domains are Sobolev extension domains. 
Proposition 5.3 Let $2 \theta \in(0,1), 1 \leq p<\infty$, and $1<q<\infty$ satisfy $2 \theta>n / q$ or, if $p=1$ let $2 \theta \geq n / q$. Then there exists a constant $C>0$ such that

$$
\left\|F_{1}\left(u_{1}, u_{2}\right)\right\|_{\mathbb{F}_{\mathcal{A}}} \leq C\left(\left\|u_{1}\right\|_{\mathbb{E}_{A_{1}}^{\text {per }}}^{2}+\left\|u_{1}\right\|_{\mathbb{E}_{A_{1}}^{\text {per }}}^{3}+\left\|u_{1}\right\|_{\mathbb{E}_{A_{1}}^{\text {per }}}\left\|u_{2}\right\|_{\mathbb{E}_{A_{2}}^{\text {per }}}\right)
$$

for all $u_{1} \in \mathbb{E}_{A_{1}}^{\text {per }}$ and $u_{2} \in \mathbb{E}_{A_{2}}^{\text {per }}$.

Proof Let $\alpha \in\{1,2,3\}$ and $\beta \in\{0,1\}$. By [2] and [17, Corollary 6.5.5], we have $D_{A}(\theta, p)=\mathrm{B}_{q, p}^{2 \theta}(\Omega)$. Lemma 5.2 implies that

$$
\left\|u_{1}^{\alpha} u_{2}^{\beta}\right\|_{L^{p}\left(0, T ; D_{A}(\theta, p)\right)} \leq C\left\|u_{1}\right\|_{L^{p}\left(0, T ; D_{A}(\theta, p)\right)}\left\|u_{1}\right\|_{L^{\infty}\left(0, T ; D_{A}(\theta, p)\right)}^{\alpha-1}\left\|u_{2}\right\|_{L^{\infty}\left(0, T ; D_{A}(\theta, p)\right)}^{\beta}
$$

for some constant $C>0$. Using that $\mathrm{W}^{1, p}\left(0, T ; \mathrm{B}_{q, p}^{2 \theta}(\Omega)\right) \subset L^{\infty}\left(0, T ; \mathrm{B}_{q, p}^{2 \theta}(\Omega)\right)$ delivers

$$
\left\|u_{1}^{\alpha} u_{2}^{\beta}\right\|_{L^{p}\left(0, T ; D_{A}(\theta, p)\right)} \leq C\left\|u_{1}\right\|_{\mathrm{W}^{1, p}\left(0, T ; D_{A}(\theta, p)\right)}^{\alpha}\left\|u_{2}\right\|_{\mathrm{W}^{1, p}\left(0, T ; D_{A}(\theta, p)\right)}^{\beta} .
$$

By definition of $\mathbb{E}_{A_{1}}^{\text {per }}$ and $\mathbb{E}_{A_{2}}^{\text {per }}$ this readily proves the proposition.

Finally, by definition of $F_{1}$ it is clear that $F_{1}(0,0)=0$. Moreover, due to the polynomial structure of $F_{1}$ it is clear that $F_{1}$ is Fréchet differentiable with $D F_{1}(0,0)=0$. Hence, we have the following proposition.

Proposition 5.4 With the definitions of this subsection the nonlinearities $F_{1}$ and $F_{2}$ satisfy Assumption N.

Now, we are ready to prove the main results presented in Sect. 4. To do so, the equilibrium points of the nonlinearities are calculated for the respective models. Afterward, the solutions to the bidomain models are written as the sum of the equilibrium solution and a perturbation. This results in an equation for the perturbation which is shown via Theorem 3.1 to have strong periodic solutions for suitable equilibrium points.

\subsection{The periodic bidomain FitzHugh-Nagumo equation}

Recall the periodic bidomain FitzHugh-Nagumo equation

$$
\begin{cases}\partial_{t} u+\varepsilon A u=I-\frac{1}{\varepsilon}\left[u^{3}-(a+1) u^{2}+a u+w\right] & \text { in } \mathbb{R} \times \Omega, \\ \partial_{t} w=c u-b w & \text { in } \mathbb{R} \times \Omega, \\ u(t)=u(t+T) & \text { in } \mathbb{R} \times \Omega, \\ w(t)=w(t+T) & \text { in } \mathbb{R} \times \Omega .\end{cases}
$$

In order to calculate the equilibrium points, we consider

$$
\begin{gathered}
u^{3}-(a+1) u^{2}+a u+w=0, \\
c u-b w=0 .
\end{gathered}
$$

Then, the equilibrium points are $\left(u_{1}, w_{1}\right)=(0,0)$ and assuming $c<b\left(\frac{(a+1)^{2}}{4}-a\right)$, we obtain furthermore 


$$
\begin{aligned}
& \left(u_{2}, w_{2}\right)=\left(\frac{1}{2}(a+1-d), \frac{c}{2 b}(a+1-d)\right), \\
& \left(u_{3}, w_{3}\right)=\left(\frac{1}{2}(a+1+d), \frac{c}{2 b}(a+1+d)\right),
\end{aligned}
$$

with $d=\sqrt{(a+1)^{2}-4\left(a+\frac{c}{b}\right)}$. In the following, we use the results from Sects. 2 and 3 to obtain periodic solutions in a neighborhood of these equilibrium points. For this purpose, we use Taylor expansion at the equilibrium points and perform the following change of variables

$$
\left(\begin{array}{l}
v \\
z
\end{array}\right):=\left(\begin{array}{c}
u-u_{\mathrm{i}} \\
w-w_{\mathrm{i}}
\end{array}\right)
$$

for $i=1,2,3$. Then, functions $F$ and $G$ describing the ionic transport defined as in the introduction read as follows

$$
\begin{aligned}
& F(v, z)=\frac{1}{\varepsilon}\left[v^{3}+\left(3 u_{\mathrm{i}}-a-1\right) v^{2}+\left(3 u_{\mathrm{i}}^{2}-2(a+1) u_{\mathrm{i}}+a\right) v+z\right], \\
& G(v, z)=-c v+b z .
\end{aligned}
$$

Plugging this into Eq. (5.4) and shifting the linear parts of $F$ and $G$ to the left-hand side yields

$$
\left\{\begin{aligned}
\partial_{t}\left(\begin{array}{l}
v \\
z
\end{array}\right)+\left(\begin{array}{c}
\varepsilon A+\frac{1}{\varepsilon}\left[3 u_{\mathrm{i}}^{2}-2(a+1) u_{\mathrm{i}}+a\right] \frac{1}{\varepsilon} \\
b
\end{array}\right)\left(\begin{array}{l}
v \\
z
\end{array}\right) & =\left(\begin{array}{c}
I-\frac{1}{\varepsilon}\left[v^{3}+\left(3 u_{\mathrm{i}}-a-1\right) v^{2}\right] \\
0
\end{array}\right), \\
v(t) & =v(t+T), \\
z(t) & =z(t+T) .
\end{aligned}\right.
$$

First of all, notice that Proposition 5.4 implies that the nonlinearity in (5.9) satisfies Assumption N. Next, regarding the system with respect to the equilibrium point $(0,0)$, then $-\left(\varepsilon A+\frac{a}{\varepsilon}\right)$ generates a bounded analytic semigroup by Proposition 4.1 and since $0 \in \rho\left(\varepsilon A+\frac{a}{\varepsilon}\right)$, we may apply Lemma 5.1 to conclude that the negative of the operator matrix in (5.9) has zero in its resolvent set and generates a bounded analytic semigroup. Consequently, Theorem 3.1 is applicable in the case of the equilibrium point $(0,0)$ and delivers a unique strong periodic solution $(v, z)$ to $(5.9)$ in the desired function space for small periodic forcings $I$.

For the second equilibrium point, we have $3 u_{2}^{2}-2(a+1) u_{2}+a<0$. Since $0 \in \sigma(A)$ the operator $-\left(\varepsilon A+\frac{1}{\varepsilon}\left[3 u_{2}^{2}-2(a+1) u_{2}+a\right]\right)$ does not generate a bounded analytic semigroup so that Lemma 5.1 is not applicable.

If

$$
u_{3}>\frac{a+1+\sqrt{(a+1)^{2}-3 a}}{3},
$$

we obtain $3 u_{3}^{2}-2(a+1) u_{3}+a>0$. Thus, $-\left(\varepsilon A+\frac{1}{\varepsilon}\left[3 u_{3}^{2}-2(a+1) u_{3}+a\right]\right)$ generates a bounded analytic semigroup by Proposition 4.1 and $0 \in \rho\left(\varepsilon A+\frac{1}{\varepsilon}\left[3 u_{3}^{2}-2(a+1) u_{3}+a\right]\right)$. Hence, we can apply Lemma 5.1 to conclude that the negative of the operator matrix in (5.9) has zero in its resolvent set and generates a bounded analytic semigroup. 
Consequently, Theorem 3.1 is applicable in this case of the equilibrium point $\left(u_{3}, w_{3}\right)$ and delivers a unique strong periodic solution $(v, z)$ to $(5.9)$ in the desired function spaces for small periodic forcings $I$. This proves Theorem 4.3 .

\subsection{The periodic bidomain Aliev-Panfilov equation}

Recall the periodic bidomain Aliev-Panfilov equation

$$
\begin{cases}\partial_{t} u+\varepsilon A u=I-\frac{1}{\varepsilon}\left[k u^{3}-k(a+1) u^{2}+k a u+u w\right] & \text { in } \mathbb{R} \times \Omega, \\ \partial_{t} w=-(k u(u-1-a)+d w) & \text { in } \mathbb{R} \times \Omega, \\ u(t)=u(t+T) & \text { in } \mathbb{R} \times \Omega, \\ w(t)=w(t+T) & \text { in } \mathbb{R} \times \Omega .\end{cases}
$$

In order to calculate the equilibrium points, we consider

$$
\begin{gathered}
k u^{3}-k(a+1) u^{2}+k a u+u w=0, \\
k u(u-1-a)+d w=0 .
\end{gathered}
$$

Then, the equilibrium points are $\left(u_{1}, w_{1}\right)=(0,0)$ and, if we assume $\frac{(a+1)^{2}}{4}+\frac{d a}{1-d}>0$, furthermore

$$
\begin{aligned}
& \left(u_{2}, w_{2}\right)=\left(\frac{a+1}{2}-e,-k u_{2}^{2}+k(a+1) u_{2}-k a\right), \\
& \left(u_{3}, w_{3}\right)=\left(\frac{a+1}{2}+e,-k u_{3}^{2}+k(a+1) u_{3}-k a\right) .
\end{aligned}
$$

with $e=\sqrt{\frac{(a+1)^{2}}{4}+\frac{d a}{1-d}}$. In the following, we want to use the results from Sects. 2 and 3 to obtain periodic solutions in a neighborhood of these equilibrium points. For this purpose, we use Taylor expansion at the equilibrium points and perform the following change of variables

$$
\left(\begin{array}{l}
v \\
z
\end{array}\right):=\left(\begin{array}{c}
u-u_{\mathrm{i}} \\
w-w_{\mathrm{i}}
\end{array}\right)
$$

for $i=1,2,3$. Then, functions $F$ and $G$ describing the ionic transport defined as in the introduction read as follows

$$
\begin{aligned}
& F(v, z)=\frac{1}{\varepsilon}\left[k v^{3}+\left(3 k u_{\mathrm{i}}-k(a+1)\right) v^{2}+\left(3 k u_{\mathrm{i}}^{2}-2 k(a+1) u_{\mathrm{i}}+k a+w_{\mathrm{i}}\right) v+u_{\mathrm{i}} z+v z\right], \\
& G(v, z)=\left(2 k u_{\mathrm{i}}-k(a+1)\right) v+d z+k v^{2} .
\end{aligned}
$$

Plugging this into Eq. (5.10) and shifting the linear parts of $F$ and $G$ to the left-hand side yields 


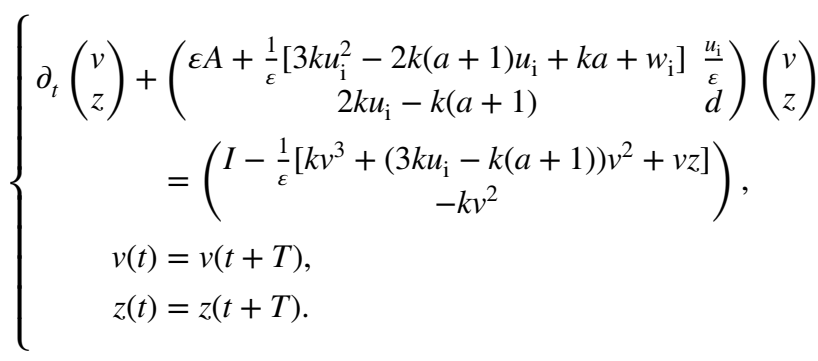

According to Proposition 5.4, the nonlinearity in (5.15) satisfies Assumption N. Moreover, considering the system for the equilibrium point $(0,0)$, then $-\left(\varepsilon A+\frac{k a}{\varepsilon}\right)$ generates a bounded analytic semigroup by Proposition 4.1 and since $0 \in \rho\left(\varepsilon A+\frac{k a}{\varepsilon}\right)$, we can apply Lemma 5.1 to conclude that the negative of the operator matrix in $(5.15)$ has zero in its resolvent set and generates a bounded analytic semigroup. Consequently, Theorem 3.1 is applicable in the case of the equilibrium point $(0,0)$ and delivers a unique strong periodic solution $(v, z)$ to $(5.15)$ in the desired function space for small periodic forcings $I$.

For the second equilibrium point, we see that $u_{2}<0$, so that the component in the upper right component of the operator matrix is negative. Therefore, we cannot apply Lemma 5.1 for $\left(u_{2}, w_{2}\right)$.

Similarly, for $\left(u_{3}, w_{3}\right)$ it is

$$
2 k u_{3}-k(a+1)=2 k e>0 .
$$

Hence, Lemma 5.1 is not applicable in this case. Altogether, Theorem 4.4 follows.

\subsection{The periodic bidomain Rogers-McCulloch equation}

The reasoning is literally the same as in Sect. 5.4 and is therefore omitted.

\subsection{The periodic bidomain Allen-Cahn equation}

Recall the periodic bidomain Allen-Cahn equation

$$
\begin{cases}\partial_{t} u+A u=I+u-u^{3} & \text { in } \mathbb{R} \times \Omega, \\ u(t)=u(t+T) & \text { in } \mathbb{R} \times \Omega .\end{cases}
$$

The equilibrium points of this system are $u_{1}=-1, u_{2}=0$, and $u_{3}=1$. In the following, we want to use the results from Sects. 2 and 3 to obtain periodic solutions in a neighborhood of these equilibrium points. For this purpose, we use Taylor expansion at the equilibrium points and perform the change of variables $v=u-u_{\mathrm{i}}$ for $i=1,2,3$. Then, the function $F(u)=u^{3}-u$ reads as follows

$$
F(v)=v^{3}+3 u_{\mathrm{i}} v^{2}-\left(1-3 u_{\mathrm{i}}^{2}\right) v, \quad i=1,2,3 .
$$

Plugging this into Eq. (5.16) and shifting the linear parts of $F$ to the left-hand side yields

$$
\begin{cases}\partial_{t} v+\left(A-1+3 u_{\mathrm{i}}^{2}\right) v=I-v^{3}-3 u_{\mathrm{i}} v^{2} & \text { in } \mathbb{R} \times \Omega, \\ u(t)=u(t+T) & \text { in } \mathbb{R} \times \Omega\end{cases}
$$


for $i=1,2,3$. According to Proposition 5.4, the nonlinearity in (5.17) satisfies Assumption N. Since $-(A+2)$ generates a bounded analytic semigroup by Proposition 4.1 and since $0 \in \rho(A+2)$, Theorem 3.1 is applicable in the case of the equilibrium points $u_{1}$ and $u_{3}$ and delivers a unique strong periodic solution $v$ to (5.17) in the desired function space for small forcings $I$. Thus, we obtain Theorem 4.6.

Acknowledgements Open Access funding provided by Projekt DEAL. We thank the referee for valuable comments concerning a previous version of this paper.

Open Access This article is licensed under a Creative Commons Attribution 4.0 International License, which permits use, sharing, adaptation, distribution and reproduction in any medium or format, as long as you give appropriate credit to the original author(s) and the source, provide a link to the Creative Commons licence, and indicate if changes were made. The images or other third party material in this article are included in the article's Creative Commons licence, unless indicated otherwise in a credit line to the material. If material is not included in the article's Creative Commons licence and your intended use is not permitted by statutory regulation or exceeds the permitted use, you will need to obtain permission directly from the copyright holder. To view a copy of this licence, visit http://creativecommons.org/licenses/by/4.0/.

\section{References}

1. Aliev, R.R., Panfilov, A.V.: A simple two-variable model of cardiac excitation. Chaos Solitons Fractals 7(3), 293-301 (1996)

2. Amann, H.: Nonhomogeneous linear and quasilinear elliptic and parabolic boundary value problems. In: Schmeisser, H., Triebel, H. (eds.) Function Spaces, Differential Operators and Nonlinear Analysis, pp. 9-126. Springer, Berlin (1993)

3. Ambrosio, L., Colli Franzone, P., Savaré, G.: On the asymptotic behaviour of anisotropic energies arising in the cardiac bidomain model. Interfaces Free Bound 2(3), 213-266 (2000)

4. Arendt, W., Bu, S.: The operator-valued Marcinkiewicz multiplier theorem and maximal regularity. Math. Z. 240(2), 311-343 (2002)

5. Bahouri, H., Chemin, J.-Y., Danchin, R.: Fourier Analysis and Nonlinear Partial Differential Equations, Grundlehren der Mathematischen Wissenschaften, vol. 343. Springer, Heidelberg (2011)

6. Bourgault, Y., Coudière, Y., Pierre, C.: Existence and uniqueness of the solution for the bidomain model used in cardiac electrophysiology. Nonlinear Anal. Real World Appl. 10(1), 458-482 (2009)

7. Colli Franzone, P., Pavarino, L.F., Scacchi, S.: Mathematical Cardiac Electrophysiology, MS\&A. Modeling, Simulation and Applications, vol. 13. Springer, Cham (2014)

8. Colli Franzone, P., Pennacchio, M., Savaré, G.: Multiscale modeling for the bioelectric activity of the heart. SIAM J. Math. Anal. 37(4), 1333-1370 (2005)

9. Franzone, P.C., Savaré, G.: Degenerate evolution systems modeling the cardiac electric field at micro- and macroscopic level. In: Lorenzi, A., Ruf, B. (eds.) Evolution Equations, Semigroups and Functional Analysis (Milano, 2000), pp. 49-78. Birkhäuser, Basel (2002)

10. Da Prato, G., Grisvard, P.: Sommes d'opérateurs linéaires et équations difféntielles opérationelles. J. Math. Pures Appl. 54(3), 305-387 (1975)

11. Denk, R., Hieber, M., Prüss, J.: R-boundedness, Fourier multipliers and problems of elliptic and parabolic type. Mem. Am. Math. Soc. 166, 788 (2003)

12. Denk, R., Hieber, M., Prüss, J.: Optimal L ${ }^{p}-\mathrm{L}^{q}$-estimates for parabolic boundary value problems with inhomogeneous data. Math. Z. 257(1), 193-224 (2007)

13. Dore, G., Venni, A.: On the closedness of the sum of two closed operators. Math. Z. 196, 189-201 (1987)

14. FitzHugh, R.: Impulses and physiological states in theoretical models of nerve membrane. Biophys. J. 1(6), 445-466 (1961)

15. Giga, Y., Kajiwara, N.: On a resolvent estimate for bidomain operators and its applications. J. Math. Anal. Appl. 459(1), 528-555 (2018)

16. Giga, Y., Kajiwara, N., Kress, K.: Strong time-periodic solutions to the bidomain equations with arbitrary large forces. Nonlinear Anal. 47, 398-413 (2019)

17. Haase, M.: The functional calculus for sectorial operators. Operator theory: advances and applications, vol. 169. Birkhäuser Verlag, Basel (2006)

18. Hieber, M., Prüss, J.: On the bidomain problem with FitzHugh-Nagumo transport. Arch. Math. 111(3), 313-327 (2018) 
19. Hieber, M., Prüss, J.: Bounded $H^{\infty}$-calculus for a class of nonlocal operators: the bidomain operator in the $L_{q}$-setting. Math. Ann. (2019). https://doi.org/10.1007/s00208-019-01916-2

20. Hytönen, T., van Neerven, J., Veraar, M., Weis, L.: Analysis in Banach Spaces, vol. II. Springer, Berlin (2017)

21. Keener, J., Sneyd, J.: Mathematical physiology. In: Fall, C.P., Marland, E.S., Wagner, J.M., Tyson, J.J. (eds.) Interdisciplinary Applied Mathematics, vol. 8. Springer, New York (1998)

22. Lunardi, A.: Analytic semigroups and optimal regularity in parabolic problems. In: Brezis, H., et al. (eds.) Progress in Nonlinear Differential Equations and their Applications, vol. 16. Birkhäuser Verlag, Basel (1995)

23. Lunardi, A.: Interpolation Theory. Lecture Notes, Scuola Normale Superiore di Pisa (New Series), 2nd edn. Edizioni della Normale, Pisa (2009)

24. Mori, Y., Matano, H.: Stability of front solutions of the bidomain equation. Commun. Pure Appl. Math. 69(12), 2364-2426 (2016)

25. Stevens, A.: Mathematics and the life-sciences: a personal point of view. Jahresber. Dtsch. Math. Ver. 119(3), 143-168 (2017)

26. Weis, L.: Operator-valued Fourier multiplier theoreems and maximal $L^{p}$-regularity. Math. Ann. 319(4), 735-758 (2001)

Publisher's Note Springer Nature remains neutral with regard to jurisdictional claims in published maps and institutional affiliations. 